\title{
A Discussion of an Analytical Per-Unit-Length Impedance Matrix Model
}

\author{
Frédéric Broydé and Evelyne Clavelier \\ Tekcem \\ 12 chemin des Hauts de Clairefontaine, 78580 Maule, France \\ e-mail: \{fredbroyde,eclavelier\}@tekcem.com
}

\author{
Daniël De Zutter and Dries Vande Ginste \\ Ghent University, Dept. of Information Technology \\ Sint-Pietersnieuwstraat 41, B-9000 Gent, Belgium \\ e-mail: \{daniel.dezutter,dries.vande.ginste\}@ugent.be
}

\begin{abstract}
An analytical model for the per-unit-length impedance matrix of a multiconductor interconnection has recently been introduced and shown to be physically reasonable. The present discussion addresses the determination of the model parameters and the model accuracy.
\end{abstract}

Keywords - Multiconductor transmission lines, resistive loss model, signal integrity analysis.

\section{INTRODUCTION}

This paper relates to an analytical model for the per-unit-length (p.u.1.) impedance matrix of a multiconductor interconnection having $n$ transmission conductors (TCs) and a reference conductor (GC), such as the structures shown in Fig. 1 and Fig. 2. The p.u.1. impedance matrix is a frequency dependent complex $n \times n$ matrix denoted by $\mathbf{Z}^{\prime}$. It may be split into two terms:

$$
\mathbf{Z}^{\prime}=\mathbf{Z}_{I}^{\prime}+j \omega \mathbf{L}_{0}^{\prime}
$$

where $\omega$ is the radian frequency, $\mathbf{L}_{0}$ is a frequency independent real $n \times n$ matrix sometimes referred to as the p.u.l. external inductance matrix, or more precisely as the high-frequency p.u.l. external inductance matrix and $\mathbf{Z}_{I}^{\prime}$ is a frequency dependent complex $n \times n$ matrix traditionally referred to as the p.u.l. internal impedance matrix. $\mathbf{L}_{0}^{\prime}$ is the p.u.l. inductance matrix computed at a non-zero frequency under the assumption that all conductors of the interconnection are ideal conductors, i.e., lossless conductors. It is usually determined using the relation $\mathbf{L}^{\prime}{ }_{0}=\mu_{0} \varepsilon_{0} \mathbf{C}_{0}^{\prime-1}$, where $\mu_{0}$ is the permeability of vacuum, $\varepsilon_{0}$ is the permittivity of vacuum and $\mathbf{C}^{\prime}{ }_{0}$ is the p.u.l. capacitance matrix of an interconnection obtained from the original interconnection by replacing the dielectrics with vacuum and the conductors with ideal conductors having the same geometry. Here, $\mathbf{Z}_{I}^{\prime}$ is defined by (1). Thus, (1) is exact and writing the imaginary part $\mathbf{Z}_{I}^{\prime}$ in the form $\operatorname{Im}\left(\mathbf{Z}_{I}^{\prime}\right)$ $=j \omega \mathbf{L}_{I}^{\prime}$ defines $\mathbf{L}_{I}^{\prime}$ as an incremental inductance instead of an internal inductance determined by the average magnetic energy within the conductors [1].

For non-magnetic conductors having a homogeneous crosssection and no surface roughness, an analytical model for $\mathbf{Z}_{I}^{\prime}$, denoted by $\mathbf{Z}_{N}^{\prime}$, has recently been proposed [2]. At high frequency (h.f.), it takes into account a normal skin effect, the crowding of currents at the edges of the conductors (edge effect), and the interaction between the current distributions on different conductors (proximity effect). It was shown that this analytical model is physically reasonable and realizable in the sense that it is accurate at $\mathrm{dc}$ and h.f., corresponds to a finite dc inductance

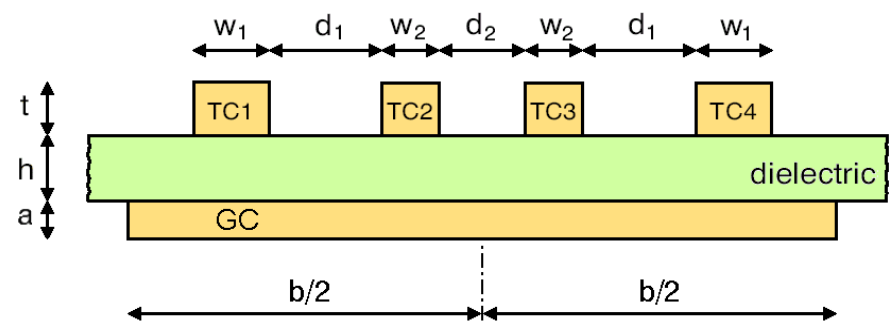

Fig. 1. Cross-section of a multiconductor microstrip interconnection comprising $n=4$ transmission conductors (TCs), identified as TC1 to TC4, and a reference or ground conductor (GC).
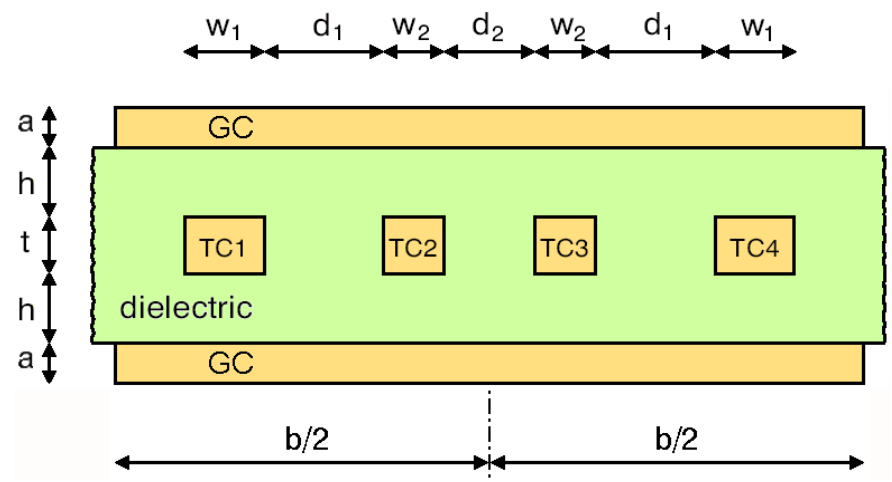

Fig. 2. Cross-section of a multiconductor stripline interconnection.

matrix, and that it represents a causal and passive linear system. Replacing $\mathbf{Z}_{I}^{\prime}$ in (1) with $\mathbf{Z}_{N}^{\prime}$ produces the analytical p.u.l. impedance matrix model discussed in this paper.

This model is briefly presented in Section II. The determination of some of the model parameters is discussed in Section III. In Section IV, we compute the model parameters for two interconnections and we compare the impedance matrix predicted by the model with an impedance matrix computed using the differential surface admittance operator technique [1] [3].

\section{Presentation OF THE $\mathbf{Z}^{\prime}{ }_{N}$ Model}

We assume that all TCs have the same homogeneous resistivity $\rho_{T C}$ and that the GC has an homogeneous resistivity $\rho_{G C}$. The matrix of the equivalent inverse widths of the TCs, denoted by $\mathbf{K}_{T C}$, and the matrix of the equivalent inverse widths of the GC, denoted by $\mathbf{K}_{G C}$, can be determined at a low computational cost [4] [5]. $\mathbf{K}_{T C}$ and $\mathbf{K}_{G C}$ are frequency independent, real and positive semidefinite $n \times n$ matrices. The $\mathbf{Z}_{N}^{\prime}$ model is defined by 


$$
\mathbf{Z}_{N}^{\prime}=\mathbf{Z}_{N R}^{\prime}+\mathbf{Z}_{N T C}^{\prime}+\mathbf{Z}_{N G C}^{\prime}
$$

where, for the indices $\alpha$ and $\beta$ ranging from 1 to $n$ with $\alpha \neq \beta$, the entries of the matrices $\mathbf{Z}_{N R}^{\prime}, \mathbf{Z}_{N T C}^{\prime}$ and $\mathbf{Z}_{N G C}^{\prime}$ are given by

$$
\begin{gathered}
\left\{\begin{array}{l}
Z_{N R \alpha \alpha}^{\prime}=R_{D C \alpha}^{\prime}+R_{D C G C}^{\prime} \\
Z_{N R \alpha \beta}^{\prime}=\frac{R_{D C G C}^{\prime}}{\sqrt{1+\frac{4 s L_{N E G G C}^{\prime 2}}{\mu_{0} \rho_{G C}\left(\max _{1 \leq i \leq n} K_{G C i i}\right)^{2}}}} \\
Z_{N T C \alpha \alpha}^{\prime}=\frac{\mu_{0} \rho_{T C} K_{T C \alpha \alpha}^{2}}{2 L_{M A X \alpha}^{\prime}}\left(\sqrt{1+\frac{4 s L_{M A X \alpha}^{\prime 2}}{\mu_{0} \rho_{T C} K_{T C \alpha \alpha}^{2}}}-1\right) \\
Z_{N T C \alpha \beta}^{\prime}=\frac{\mu_{0} \rho_{T C} K_{T C \alpha \beta}\left(\sqrt{1+\frac{4 s}{\mu_{0} \rho_{T C}}\left(\min \left\{\frac{L_{M A X \alpha}^{\prime}}{K_{T C \alpha \alpha}}, \frac{L_{M A X \beta}^{\prime}}{K_{T C \beta \beta}}\right\}\right)^{2}}-1\right)}{2 \min \left\{\frac{L_{M A X \alpha}^{\prime}}{K_{T C \alpha \alpha}}, \frac{L_{M A X \beta}^{\prime}}{K_{T C \beta \beta}}\right\}}
\end{array}\right.
\end{gathered}
$$

and

$$
\mathbf{Z}_{N G C}^{\prime}=\frac{\mu_{0} \rho_{G C} \max _{1 \leq i \leq n} K_{G C i i}}{2 L_{M A X G C}^{\prime}}\left(\sqrt{1+\frac{4 s L_{M A X G C}^{\prime 2}}{\mu_{0} \rho_{G C}\left(\max _{1 \leq i \leq n} K_{G C i i}\right)^{2}}}-1\right) \mathbf{K}_{G C}
$$

where $s$ is the Laplace transform variable, where each square root symbol denotes the principal root, where the p.u.l. dc resistances of the TCs are denoted by $R_{D C 1}^{\prime}$ to $R_{D C n}^{\prime}$, and where the p.u.l. resistance $R_{D C G C}^{\prime}$ and the p.u.l. inductances $L_{M A X 1}^{\prime}$ to $L_{M A X n}^{\prime}$, $L_{N E G G C}^{\prime}$ and $L_{M A X G C}^{\prime}$ are positive parameters.

At frequencies high enough for the skin effect to be well developed, the largest contribution to $\mathbf{Z}_{N}^{\prime}$ comes from $\mathbf{Z}_{N T C}^{\prime}$ and $\mathbf{Z}_{N G C}^{\prime}$ and the 1 are negligible in (4) and (5). Thus, the parameters controlling the h.f. behavior of the model are $\rho_{T C}, \rho_{G C}, \mathbf{K}_{T C}$ and $\mathbf{K}_{G C}$. Conversely, the de value of $\mathbf{Z}_{N}^{\prime}$ is a resistance matrix solely determined by $\mathbf{Z}_{N R}^{\prime}$. It may be easily verified that $\mathbf{Z}_{N R}^{\prime}, \mathbf{Z}_{N T C}^{\prime}$ and $\mathbf{Z}_{N G C}^{\prime}$ contribute to the dc inductance matrix. The main motivation for introducing the $\mathbf{Z}_{N}^{\prime}$ model is that all entries of the dc inductance matrix are finite, unlike the dc inductance matrix of a model in the form $\mathbf{Z}_{S}^{\prime}=\mathbf{A}+s^{1 / 2} \mathbf{B}$ where $\mathbf{A}$ and $\mathbf{B}$ are two frequency independent matrices. The parameters $R_{D C 1}^{\prime}$ to $R_{D C n}^{\prime}$ of $\mathbf{Z}_{N R}^{\prime}$ are unambiguously defined above, but not the parameters $R_{D C G C}^{\prime}, L_{M A X 1}^{\prime}{ }_{M A X} L_{M A X}^{\prime}, L_{N E G G C}^{\prime}$ and $L_{M A X G C}^{\prime}$. They will be further discussed in the Section III and IV.

It was observed that $\mathbf{K}_{T C}$ is strictly diagonally dominant. Using this result, it was proved that the impedance matrices $\mathbf{Z}_{N}^{\prime}, \mathbf{Z}_{N R}^{\prime}$, $\mathbf{Z}_{N T C}^{\prime}$ and $\mathbf{Z}_{N G C}^{\prime}$ each represents a passive linear system, and consequently a causal system [2]. In [2], it was assumed that $L_{N E G G C}^{\prime}=L_{M A X G C}^{\prime}$, but this assumption has been removed in (3). This has no impact on the passivity of $\mathbf{Z}_{N}^{\prime}, \mathbf{Z}_{N R}^{\prime}, \mathbf{Z}_{N T C}^{\prime}$ and $\mathbf{Z}_{N G C}^{\prime}$. $L_{N E G G C}^{\prime}$ controls a possible decrease of low frequency mutual impedances, caused by the proximity effect.

\section{Discussion OF SOME MOdEL PARAMETERS}

Let $\mathbf{L}_{D C}^{\prime}$ be the dc inductance matrix of the interconnection. In [6] it is shown that, for a multiconductor microstrip structure such as the one shown in Fig. 1, all entries of $\mathbf{L}_{D C}^{\prime}$ become large as $b \rightarrow \infty$. Using [7, § 4], it can be shown that the same applies to a multiconductor stripline structure such as the one shown in Fig. 2. In contrast, if the breadth $b$ of the GC exceeds the total width $W$ occupied by the TCs and the spacing between them, $\mathbf{L}_{0}$ quickly approaches a limit as $b$ becomes large.

As an example, the entries of $\mathbf{L}_{D C}^{\prime}$ and $\mathbf{L}_{0}$ are shown in Fig. 3 as a function of $b$, for the multiconductor microstrip interconnection shown in Fig. 1, in the case $t=h=a=w_{1}=w_{2}=$ $d_{1}=d_{2}=50 \mu \mathrm{m}$. $\mathbf{L}_{D C}^{\prime}$ was computed using an exact formula implementing modified partial inductances [6]. All entries of $\mathbf{L}_{D C}^{\prime}$ are equivalent to $\left(\mu_{0} \ln b\right) /(2 \pi)$ as $b \rightarrow \infty$, so that, in Fig. 3, they increase slowly as $b$ increases. $\mathbf{L}_{0}$ was computed by the method of moment using pulse expansion and 860 matching points.

The fact that the entries of $\mathbf{L}_{D C}^{\prime}$ become large as $b \rightarrow \infty$ follows from an homogeneous dc current distribution in the GC, for which most of the current in the GC flows at a distance of the TC which increases with $b$. We are led to the following statements:

- for $b>W+3(h+t)=650 \mu \mathrm{m}$, the h.f. current distribution in the $\mathrm{GC}$ is close to the current distribution for $b=\infty$, so that we make a small error if we use $\mathbf{L}_{0}^{\prime}, \mathbf{K}_{T C}$ and $\mathbf{K}_{G C}$ computed for $b=\infty$; - $L_{M A X 1}^{\prime}$ to $L_{M A X n}^{\prime}, L_{N E G G C}^{\prime}$ and $L_{M A X G C}^{\prime}$ must be determined for

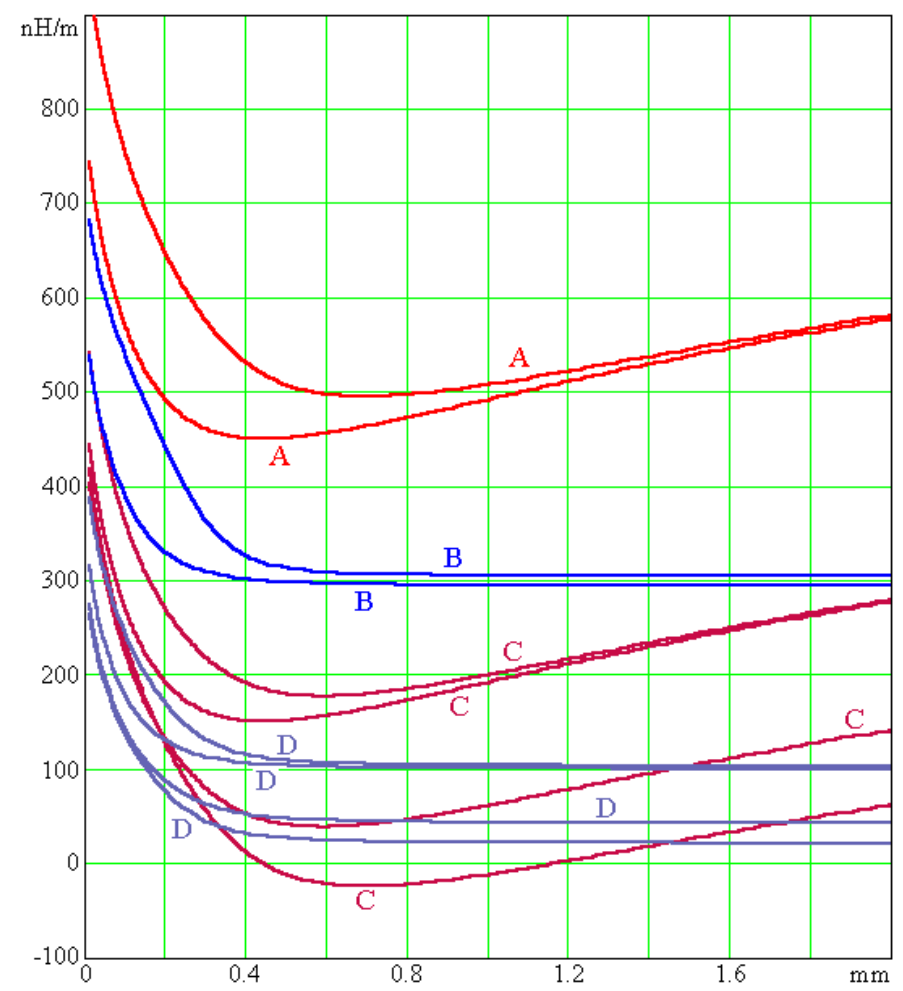

Fig. 3. For the multiconductor microstrip interconnection, the dc p.u.l. self-inductances ( 2 curves $A$ ), the diagonal entries of $L_{0}(2$ curves B), the dc p.u.I. mutual inductances (4 curves $C$ ) and the non-diagonal entries of $\mathbf{L}_{0}(4$ curves $\mathrm{D})$ as a function of $b$. 


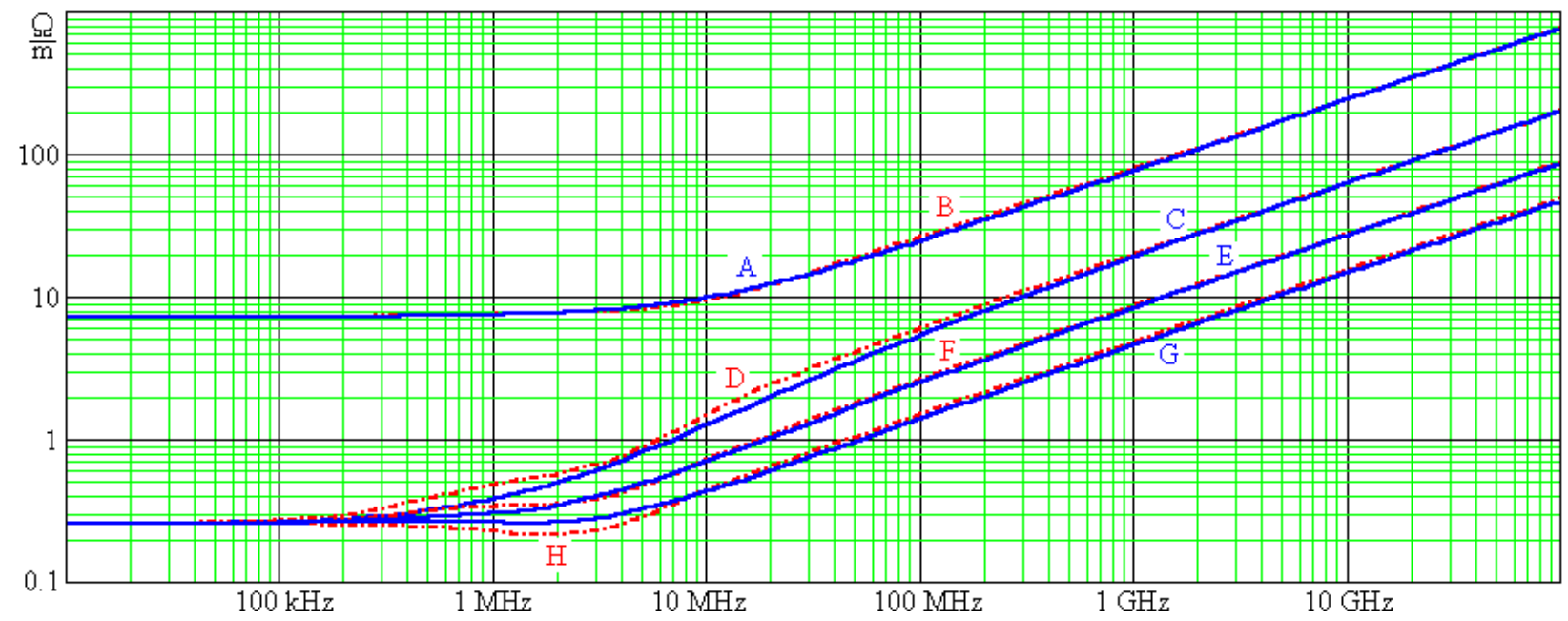

Fig. 4. For the multiconductor microstrip structure, some entries of $\mathbf{R}^{\prime}$ according to the $\mathbf{Z}^{\prime}{ }_{N}$ model (blue solid curves) and to the accurate computation (red dadot curves): $R^{\prime}{ }_{11}(\mathrm{~A}$ and $\mathrm{B}), R^{\prime}{ }_{12}(\mathrm{C}$ and $\mathrm{D}), R^{\prime}{ }_{13}(\mathrm{E}$ and $\mathrm{F})$ and $R^{\prime}{ }_{14}(\mathrm{G}$ and $\mathrm{H})$.

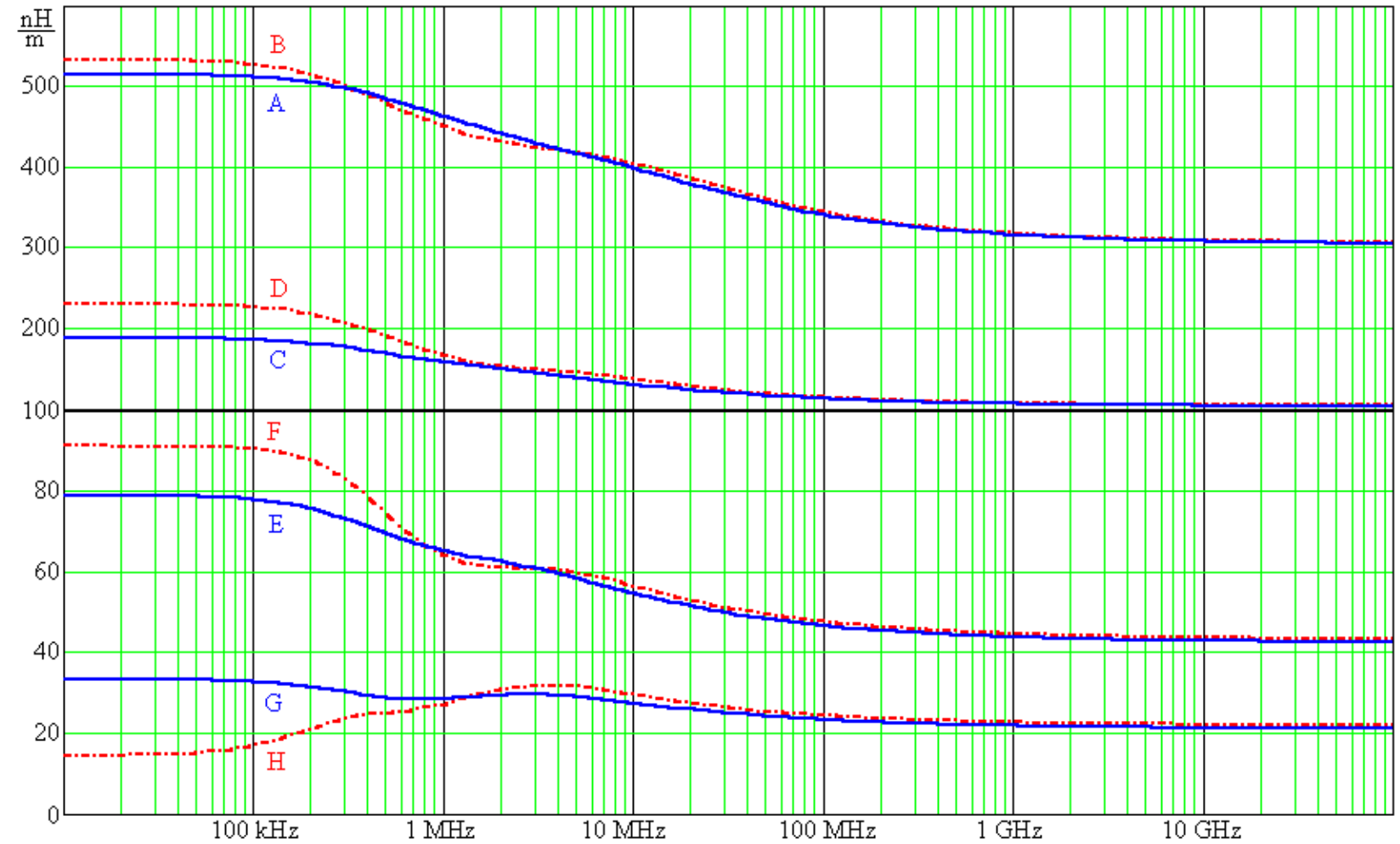

Fig. 5. For the multiconductor microstrip structure, some entries of $L^{\prime}$ according to the $\mathbf{Z}_{N}^{\prime}$ model (blue solid curves) and to the accurate computation (red dadot curves): $L^{\prime}{ }_{11}(A$ and $B), L^{\prime}{ }_{12}(C$ and $D), L_{13}^{\prime}(E$ and $F)$ and $L^{\prime}{ }_{14}(G$ and $H)$.

a finite value of $b$, for the main objective of minimizing the error on the diagonal entries $\mathbf{R}^{\prime}=\operatorname{Re}\left(\mathbf{Z}^{\prime}\right)$ at intermediate frequencies; - $R_{D C G C}^{\prime}$ should be the actual dc resistance of the GC.

\section{TWO EXAMPLES}

A first example relates to the multiconductor microstrip interconnection defined above, in the case $b=1350 \mu \mathrm{m}$. In Fig. 3, we see that all entries of $\mathbf{L}_{D C}^{\prime}$ are positive in this case. Fig. 4 and Fig. 5 show some entries of $\mathbf{R}^{\prime}$ and $\mathbf{L}^{\prime}=\operatorname{Im}\left(\mathbf{Z}^{\prime}\right) / \omega$ according to the
$\mathbf{Z}_{N}^{\prime}$ model and to an accurate numerical computation using the differential surface admittance operator technique [1] [3]. For $\mathbf{R}^{\prime}$, we observe a good agreement at every frequency and an excellent agreement at every frequency except in the intermediate frequency range from $200 \mathrm{kHz}$ to $100 \mathrm{MHz}$. For $\mathbf{L}$ ', we observe a good agreement except at frequencies below $500 \mathrm{kHz}$, but this discrepancy cannot lead to a significant error in a simulation involving an on-board, on-package, or on-chip interconnections.

A second example relates to the multiconductor stripline 


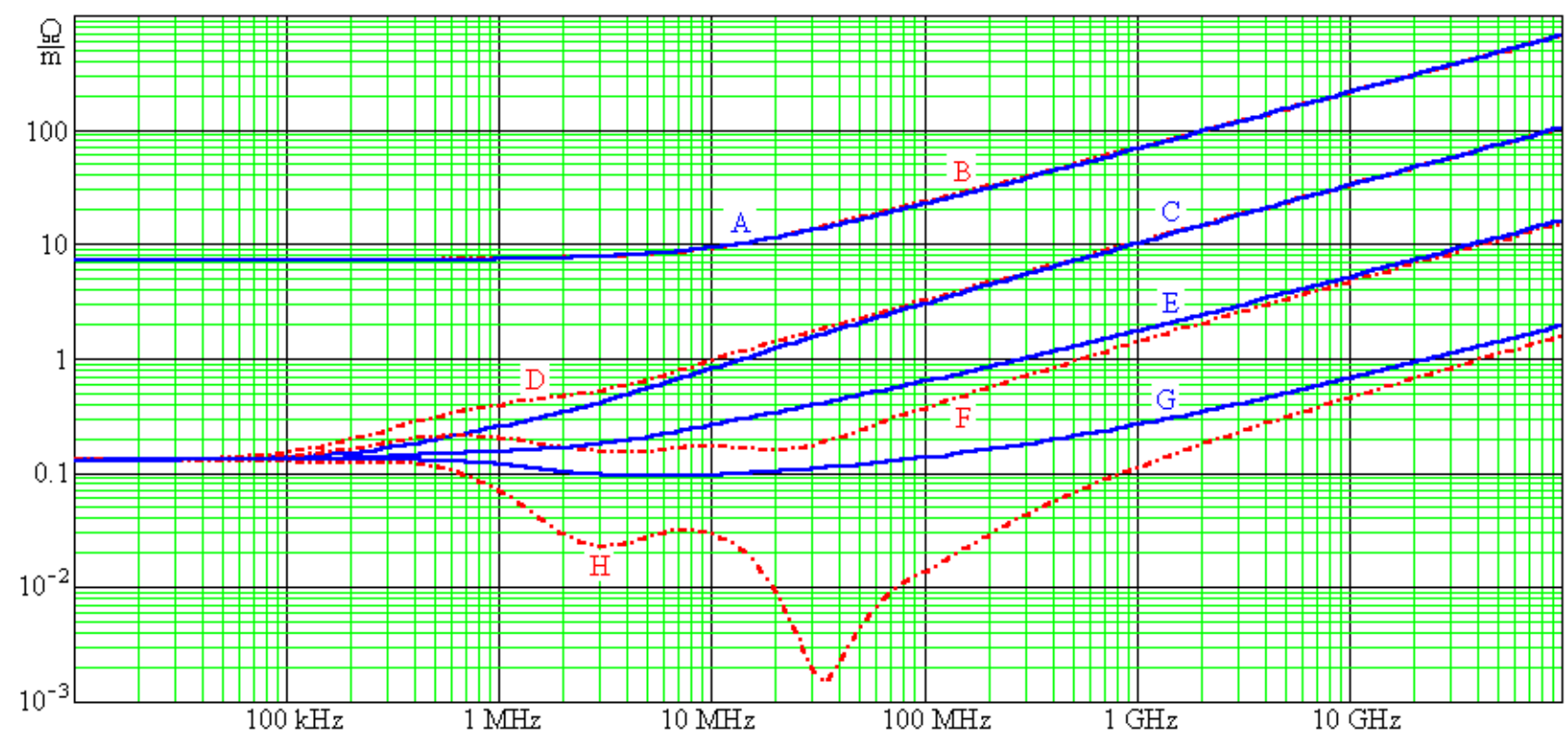

Fig. 6. For the multiconductor stripline structure, some entries of $\mathbf{R}^{\prime}$ according to the $\mathbf{Z}^{\prime}{ }_{N}$ model (blue solid curves) and to the accurate computation (red dadot curves): $R^{\prime}{ }_{11}(\mathrm{~A}$ and $\mathrm{B}), R^{\prime}{ }_{12}(\mathrm{C}$ and $\mathrm{D}), R_{13}^{\prime}(\mathrm{E}$ and $\mathrm{F})$ and $R^{\prime}{ }_{14}(\mathrm{G}$ and $\mathrm{H})$.

interconnection shown in Fig. 2, in the case $t=h=a=w_{1}=w_{2}=$ $d_{1}=d_{2}=50 \mu \mathrm{m}$ and $b=1350 \mu \mathrm{m}$. Here all entries of $\mathbf{L}_{D C}^{\prime}$ are positive, except $\mathbf{L}_{D C 14}^{\prime}$ and $\mathbf{L}_{D C 41}^{\prime}$. Fig. 6 shows some entries of $\mathbf{R}^{\prime}$ according to the $\mathbf{Z}_{N}^{\prime}$ model and to the same numerical computation as above. The numerical model predicts a nonincreasing frequency dependence for $R^{\prime}{ }_{14}$, below about $35 \mathrm{MHz}$. Since such a complicated behavior is not described in the literature, we performed an independent verification using a 2-D code based on the method of filaments, up to $10 \mathrm{MHz}$, for which we obtained the same result. A good agreement between the $\mathbf{Z}_{N}^{\prime}$ models and the numerical computation is obtained only for the diagonal entries of $\mathbf{R}^{\prime}$ and for the non-diagonal entries of $\mathbf{R}^{\prime}$ exceeding about $2 \Omega / \mathrm{m}$. However, the error observed for the nondiagonal entries of $\mathbf{R}^{\prime}$ corresponds to negligible losses compared to the losses caused by the diagonal entry of $\mathbf{R}^{\prime}$.

In both examples, at every frequency, the maximum column sum matrix norm and the maximum row sum matrix norm of the error in $\mathbf{R}^{\prime}$ are small compared to the corresponding norm of $\mathbf{R}^{\prime}$, and very small except in an intermediate frequency range.

In both examples, the $\mathbf{Z}_{N}^{\prime}$ model uses $\mathbf{L}^{\prime}{ }_{0}, \mathbf{K}_{T C}$ and $\mathbf{K}_{G C}$ given in $\S$ VII of [5], computed for $b=\infty$. It uses the values of $L_{M A X 1}^{\prime}$ to $L_{M A X n}^{\prime}, L_{N E G G C}^{\prime}$ and $L_{M A X G C}^{\prime}$ produced by an optimization process in which the objective function is a linear combination of squared errors of entries of $\mathbf{R}^{\prime}$ at different frequencies. For Fig. 4 and Fig. 5, we have used $L_{M A X 1}^{\prime}=L_{M A X}^{\prime}{ }^{\prime} \approx 76.38 \mathrm{nH} / \mathrm{m}$, $L_{M A X 2}^{\prime}=L_{M A X 3}^{\prime} \approx 88.00 \mathrm{nH} / \mathrm{m}, L_{N E G G C}^{\prime} \approx 67.57 \mathrm{nH} / \mathrm{m}$ and $L_{M A X G C}^{\prime} \approx 134.28 \mathrm{nH} / \mathrm{m}$. For Fig. 6 , we have used $L_{M A X 1}^{\prime}=$ $L_{M A X 4}^{\prime} \approx 64.73 \mathrm{nH} / \mathrm{m}, L_{M A X 2}^{\prime}=L_{M A X 3}^{\prime} \approx 71.35 \mathrm{nH} / \mathrm{m}, L_{N E G G C}^{\prime} \approx$ $55.21 \mathrm{nH} / \mathrm{m}$ and $L_{M A X G C}^{\prime} \approx 146.99 \mathrm{nH} / \mathrm{m}$.

\section{CONCLUSION}

We have used an analytical and physically reasonable p.u.l. impedance matrix model of a multiconductor interconnection, presenting two differences with the approach used in [2]: a new parameter has been introduced and a least square error optimization was used to obtain $L_{M A X 1}^{\prime}$ to $L_{M A X n}^{\prime}, L_{N E G G C}^{\prime}$ and $L_{M A X G C}^{\prime}$. The values obtained for $L_{M A X 1}^{\prime}$ to $L_{M A X n}^{\prime}$ and $L_{M A X G C}^{\prime}$ show that they cannot be regarded as de internal inductances, which are less than $48.32 \mathrm{nH} / \mathrm{m}$ for any rectangular conductor [8].

The $\mathbf{Z}_{N}^{\prime}$ model used in this paper has a small number of real and frequency independent parameters, but it provides a good accuracy for the types of interconnections that we have considered.

\section{REFERENCES}

[1] T. Demeester, D. De Zutter, "Internal Impedance of Composite Conductors with Arbitrary Cross Section", IEEE Trans. Electromagn. Compat., vol. 51, No. 1, February 2009, pp. 101-107.

[2] F. Broydé, E. Clavelier, "An Analytical Resistive Loss Model for Multiconductor Transmission Lines and the Proof of its Passivity", Proc. IEEE $20^{\text {th }}$ Conference on Electrical Performance of Electronic Packaging and Systems, EPEPS 2011, San Jose, pp. 119-122, Oct. 23-26, 2011.

[3] D. De Zutter, L. Knockaert, "Skin Effect Modeling Based on a Differential Surface Admittance Operator", IEEE Trans. Microwave Theory Tech., Vol. 53, No. 8, pp. 2526-2538, August 2005.

[4] F. Broydé, E. Clavelier, "A Simple Computation of the High-Frequency PerUnit-Length Resistance Matrix”, Proc. of the 15th IEEE Workshop on Signal Propagation on Interconnects, SPI 2011, May 2011, pp. 121-124.

[5] F. Broydé, E. Clavelier, "A Computation of the High-Frequency Per-UnitLength Resistance Matrix of a Multiconductor Interconnections", Proc. of the 10th Int. Symp. on Electromagnetic Compatibility, EMC Europe 2011, York, UK, pp. 351-356, Sept. 26-30, 2011.

[6] F. Broydé, E. Clavelier, L. Broydé, “A Direct Current Per-Unit-Length Inductance Matrix Computation Using Modified Partial Inductances”, Proc. CEM 2012 Int. Symp. and Exhibition on Electromagnetic Compatibility (16ème coll. int. et exposition sur la compatibilité électromagnétique), Rouen, April 2012. Available: http://www.eurexcem.com/bibliodef.htm.

[7] C. Hoer, C. Love, "Exact Inductance Equations for Rectangular Conductors With Applications to More Complicated Geometries", J. Res. Nat. Bur. Stand., Vol. 69C, No. 2, pp. 127-137, April-June 1965.

[8] R. De Smedt, "Addition to «DC Internal Inductance for a Conductor of Rectangular Cross Section»", IEEE Trans. Electromagn. Compat., vol. 51, No. 3, August 2009, pp. 875-876. 